\title{
A Graphical Pressure Sensor Array With Multilayered Structure: Written Through Graphene Ink on Cellulose Paper Substrate
}

Chao Ji

Taiyuan University of Technology

Qiang Zhang

Taiyuan University of Technology

Qiang Li

Taiyuan University of Technology

Zhen Pei

Taiyuan University of Technology

Dong Zhao

Taiyuan University of Technology

Zhongyun Yuan

Taiyuan University of Technology

Jie Wang

Taiyuan University of Technology

Wendong Zhang

Taiyuan University of Technology

Shengbo Sang ( $\nabla$ sunboa-sang@tyut.edu.cn )

Taiyuan University of Technology

\section{Research Article}

Keywords: Paper-based sensor, Multilayered structure, Graphical sensor array, Safety warning,

Piezoresistive pressure sensor

Posted Date: July 27th, 2021

DOl: https://doi.org/10.21203/rs.3.rs-713142/v1

License: (c) (1) This work is licensed under a Creative Commons Attribution 4.0 International License.

Read Full License 
A Graphical Pressure Sensor Array with Multilayered Structure: Written through

\section{Graphene Ink on Cellulose Paper Substrate}

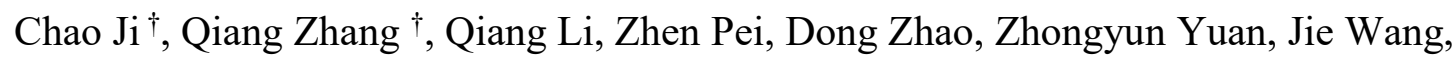
Wendong Zhang, Shengbo Sang*

MicroNano System Research Center, Key Laboratory of Advanced Transducers and Intelligent Control System of the Ministry of Education and Shanxi Province, College of Information and Computer, Taiyuan University of Technology, Taiyuan 030024, Shanxi,

\section{China}

$\dagger$ These authors contributed equally to this work

*E-mail: sunboa-sang@tyut.edu.cn

\section{Abstract}

Paper based flexible pressure sensors have received extensive attention due to their recoverability and accessibility. In this paper, we proposed graphical pressure sensors array with multilayered structure. A simple writing method was adopted to achieve the adsorption of sensitive materials on the fiber structure of cellulose paper. Pressure sensors with 1, 3, 5 and 7 stacked layers were fabricated and compared, respectively. The results show that the 7layers sensor combined high sensitivity $\left(44 \mathrm{kPa}^{-1}\right)$ and fast time response $(150 \mathrm{~ms})$. The highly sensitive stacked paper-based sensor array realizes the pressure detection of objects and special-shaped surfaces. A pressure sensor base on commercial corrugated box was also fabricated to compare. The corrugated carton array was made to switch reminder devices for its convenience and accessibility. Since there are many scenarios that require a safe distance, especially under the influence of the COVID-19, the writable paper-based sensor array was used to realize graphical distance perception and warning.

Keyword : Paper-based sensor; Multilayered structure; Graphical sensor array; Safety warning; Piezoresistive pressure sensor 


\section{Introduction}

Flexible pressure sensor, a kind of device based on flexible substrate and sensitive material to detect the applied force, is widely used in health monitoring[1-5], motion detection[6-8] and robotic skin [9-12] owing to their flexibility and real-time detecting capability. The environmentally friendly pressure sensors are an important research direction at present. However, most of the flexible pressure sensors substrates are polymers [13-19], which are difficult to recycle or degrade.

Paper, as a easily available, degradable and inexpensive material, has been increasingly used in pressure detection [20-24]. Yang et al. [25] used tissue paper saturated with poly(3,4ethylenedioxythiophene) polystyrene sulfonate (PEDOT: PSS) to construct pressure sensors, exhibiting an enhanced response when the active material is folded or stacked. Guo et al. [26] provided a method using sandwiching porous MXene-impregnated tissue paper between a biodegradable polylactic acid (PLA) thin sheet and an interdigitated electrodecoated PLA thin sheet to prepare the highly sensitive pressure sensors. Tao et al. [27] researched a graphene-paper pressure sensor using laminated structure, which can be applied in pulse detection, respiratory detection, voice recognition. In addition, the paper-based flexible devices can also be prepared by mixed with nanomaterials in paper-making process [28-30] or high temperature pyrolysis [31]. However, easy processing methods to prepare allpaper based pressure sensors with high sensitivity still need to research.

Handwriting, which using pencils [32-34] or ink pens [35-40], is a simple, fast, low-cost and graphical method to create circuits on paper. Costa et al. [41] prepared a system include a pencil-written graphite force-sensitive resistor, a pencil-drawn RC filter, a pen-written halfwave rectifier, and a commercial front-end voltage amplifier. The sensitivity of the pressure sensor by writing has yet to be improved, which the multilayer structure can achieve. The use of writing for multilayer structure design requires that nanomaterial ink can form conductive channels between the layers. The diameter of the nanomaterial is a key issue that needs to be 
considered. If the diameter is too large, it is difficult to pass through the fiber structure, and it is difficult to conduct, which affects the conductivity. Meanwhile, if the diameter is too little, material rarely remaining in the intermediate layer also affect conductivity. Therefore, we choose graphene, a two-dimensional nanomaterial with a moderate size, as the ink, which can pass through the fiber structure and stay in the middle layer to form a conductive path.

In this paper, we proposed a simple writing method to prepare patterned multilayer paper-based pressure sensors to improve the sensitivity. Graphene was choose as the ink material and porous cellulose paper as the substrate. Paper-based sensors with different layers were prepared to analyze the effect of the number of layers on the sensitivity. Corrugated carton was chosen to compare the performance of different multi-layer structures. Due to the cardboard carton structure was not easy to write, the soaking method was used for preparation. The performance of multi-layer cellulose paper and corrugated carton were compared in terms of sensitivity, test range and response time. These sensors were applied in different scenarios for safety monitoring, providing a new direction for the application of paper-based pressure sensors.

\section{Experimental section}

\subsection{Preparation of pressure sensor based on corrugated box}

Disperse $100 \mathrm{mg}$ of graphene ultrasonically in $50 \mathrm{ml}$ of alcohol to form a uniform graphene dispersion, and then divide the dispersion into two bottles. Cut the corrugated boxes into squares with a surface area of $1 \mathrm{~cm} * 1 \mathrm{~cm}$, soak them in the graphene dispersion, then place them in a petri dish and heat them on a heating plate at $50{ }^{\circ} \mathrm{C}$ for 10 minutes, and then repeat the soaking of these corrugated carton after drying. Repeat 3 times to make the graphene adhere to the corrugated carton.

\subsection{Preparation of multi-layer cellulose paper pressure sensor}

Dip the writing brush into the graphene dispersion and write on the cellulose paper. Use the brush to write back and forth on the cellulose paper, coat the graphene dispersion evenly 
on the paper, put it in a petri dish and heat it on a heating plate at $50^{\circ} \mathrm{C}$ for 5 minutes, and repeat after drying. Write with a brush, repeat 3 times to make the graphene adhere to the cellulose paper, and then cut the graphene-attached cellulose paper into paper sheets with a surface area of $1 \mathrm{~cm}^{*} 1 \mathrm{~cm}$. The multi-layer cellulose paper pressure sensor is fixed and separated by double-sided tape, so that the layers are connected with each other and have gaps.

\subsection{Electrode printing and device preparation}

Place the conductive silver glue in the needle tube of the dispenser, and print a single pressure sensor electrode and a $2 * 2$ array pressure sensor electrode according to the interdigital pattern in Figure 1, and print it on a blank cellulose paper. The use of coplanar cross-fingered electrodes can avoid the influence of tangential forces when applying pressure. When it is not dry, put the prepared graphene/different layers of cellulose paper and corrugated carton on the electrode, and use the adhesive of silver glue to connect the upper sensor with the lower electrode. Attach tape to the top layer of the device to insulate the sensor from the outside. The preparation flow chart is shown in Figure 1.

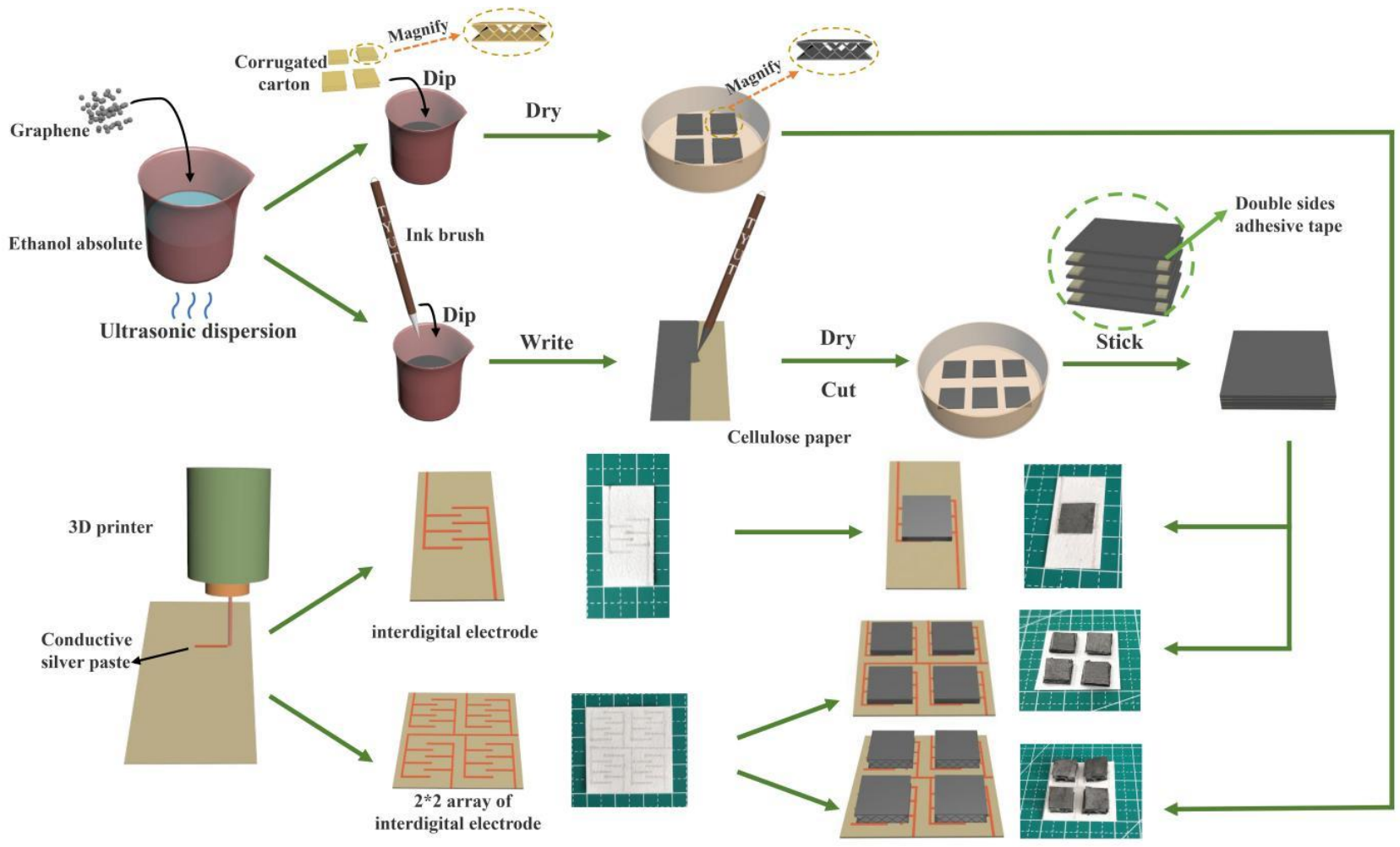




\section{Results and discussion}

Figure 2a showed the SEM characterization of cellulose paper, illustrating the porous structure, while the size of smaller aperture was $15 \mu \mathrm{m}$ and the larger was $40 \mu \mathrm{m}$. Figure $2 \mathrm{~b}$ showed the SEM characterization of cellulose paper written by graphene. It can be seen that the graphene covered the fiber uniformly, and the size of the graphene was about $10 \mu \mathrm{m}$, which was smaller than the pores bewteen the fibers from a larger version in Figure 2c. Therefore, graphene can pass through the pores between the fibers from top to bottom to form a conductive layer. The results showed that the choice of graphene as the ink for the filter paper substrate plays an important role in the preparation of multi-layer structure pressure sensors. Figure $2 \mathrm{~d}$ showed the SEM characterization of corrugated carton, and can also be seen that there are relatively compact porous (approximately $15 \mu \mathrm{m}$ ) structures. Figure $2 \mathrm{e}$ is the SEM characterization of the surface of the corrugated carton soaking graphene, and Figure $2 \mathrm{f}$ is the larger version, explaining the specific state of graphene dispersion. The distribution of graphene on the surface of the box was not distributed on cellulose paper. The uniformity indicated that writing can make graphene more evenly distributed on the surface of the substrate compared with soaking, explaining the importance of writing method for the paper substrate and ink adaptability and matching selection simultaneously.

(a)

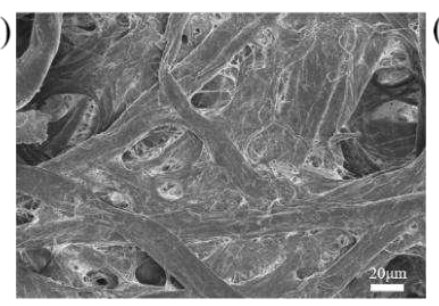

(d)

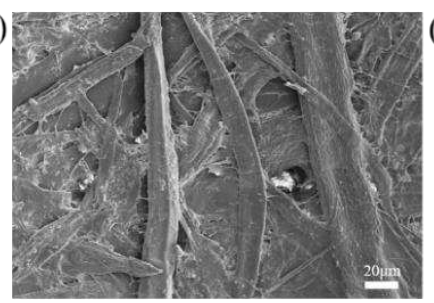

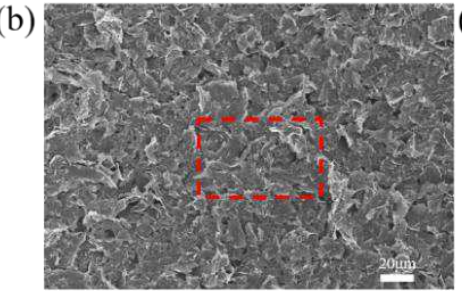

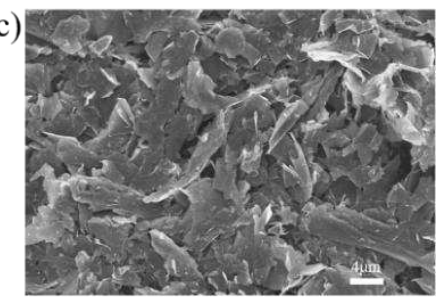

(e)

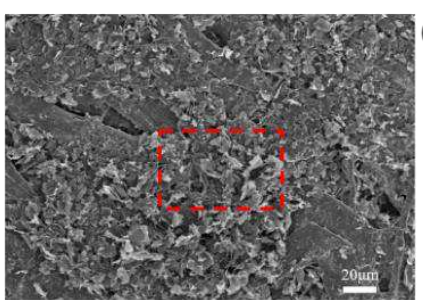

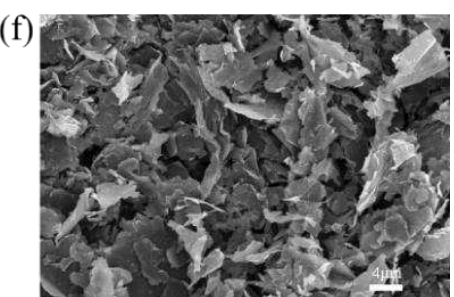
version of (b), (d) corrugated carton, (e)graphene/corrugated carton, (f) larger version of (e). 
Figure 3a showed the pressure response test of graphene-different layers of cellulose paper/corrugated carton. The sensitivity of the 1-layer cellulose paper-based sensor was relatively small, $6 \mathrm{kPa}^{-1}$, and the response range was 0-39 $\mathrm{kPa}$. With the increase in the number of layers, the sensitivity became larger and the response range became smaller. The sensitivity of the 7-layers cellulose paper-based sensor was $44 \mathrm{kPa}^{-1}$, and the response range is small pressure, the sensor with more layers would cause more contact between the layers, which lead to the resistance change greater. If the cellulose paper had more than 7 layers, the

0-4 kPa. Double-sided tape was used to separate different layers. More layers made more gaps, which led to increase the initial resistance. Therefore, when the sensor was subjected to a sensor will be unstable. When pressure was under applied force, the device would be affected by the tangential force caused by instability. The sensitivity of the corrugated cardboard box with graphene was $8 \mathrm{kPa}^{-1}$, and the response range was $0-10 \mathrm{kPa}$. Owing to the hard texture of the corrugated carton and hardness of the corrugated connection, the deformation of the carton is smaller under same pressure, which results in lower sensitivity. Figure $3 \mathrm{~d}$ showed the sensitivity and response range of different layers of cellulose paper/corrugated carton. Among different sensors using multilayer cellulose paper, the 7-layers device had the highest sensitivity, and was selected for subsequent testing and application.

Figure $3 b$ and $3 c$ were the response time of cellulose paper with 7 layers and corrugated carton under a pressure of $2 \mathrm{kPa}$. The response time of 7-layers cellulose paper was $150 \mathrm{~ms}$, and the corrugated box was $270 \mathrm{~ms}$. The reason why corrugated boxes respond more slowly than 7-layers cellulose paper was that the stiffer texture of corrugated boxes may affect the response speed. Figure 3e was the repeatability test under different pressures for the 7-layers paper-based sensor. Figure $3 \mathrm{f}$ shows the results of 200 repeated tests at a pressure of $1 \mathrm{kPa}$. It can be seen that the sensor had stable repeatability. 

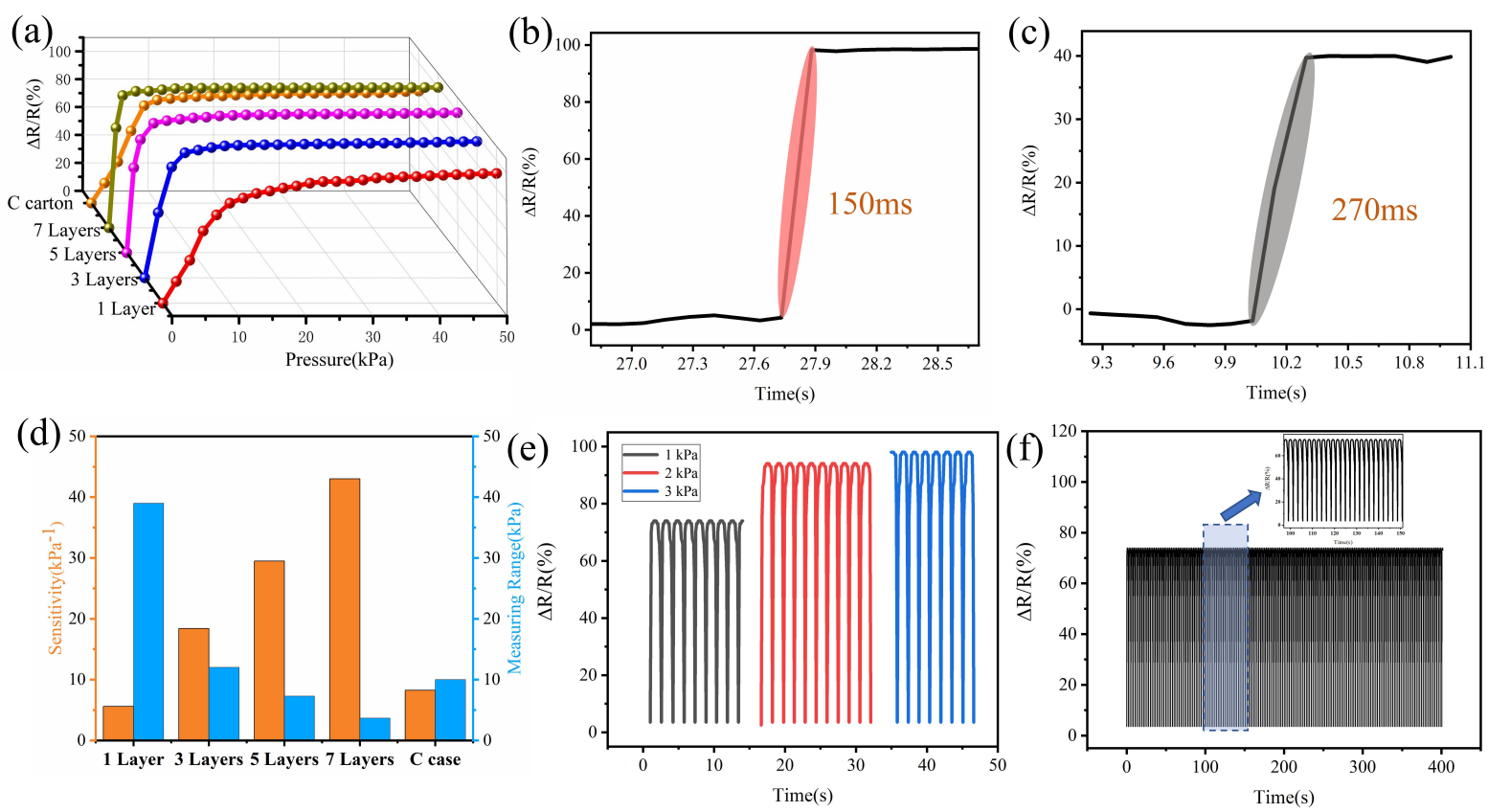

Figure 3. (a) The pressure response test of graphene-different layers of cellulose paper/corrugated carton. (b) Sensitivity and response range of graphene-different layers of cellulose paper/corrugated carton. Response time test results of (c) 7-layers cellulose paper and

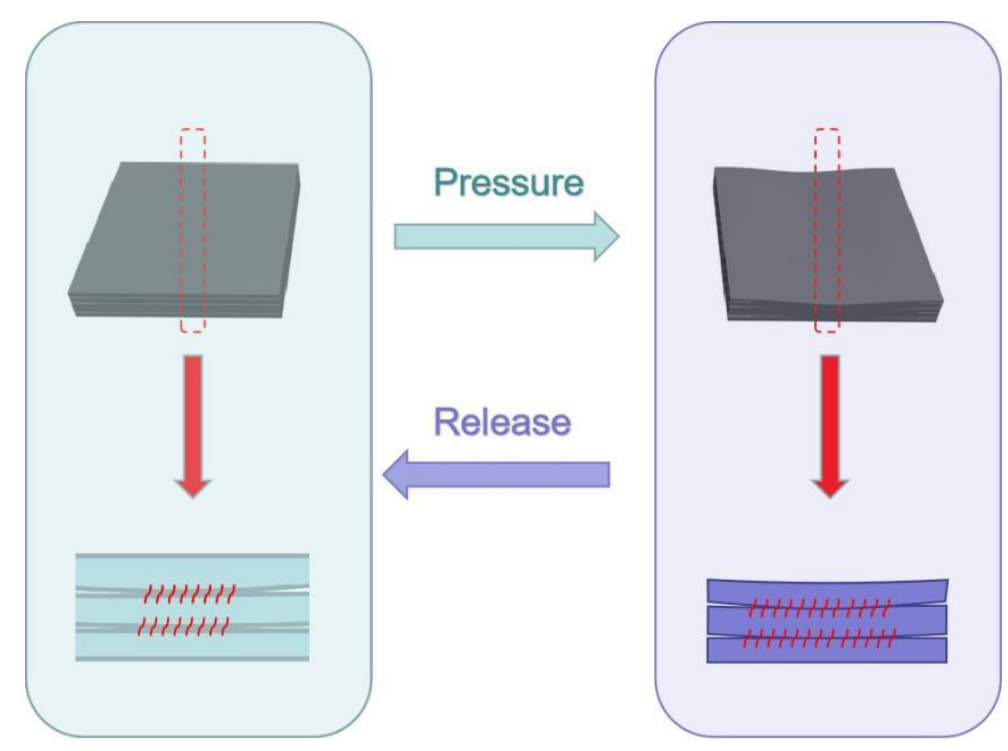

Figure 4. Paper based pressure sensor schematic diagram

Figure 4 was the schematic diagram of the paper-based pressure sensor. When the pressure was applied, there are certain pores between different layers of paper base, which are connected with each other. When the pressure is applied, the pores between layers decrease 
and the area of mutual contact increases, so that the conductive path between the paper is connected and the resistance becomes smaller. After releasing the pressure, the interlayer pores are reduced and the resistance is restored.

Figure 5 showed a $2 \times 2$ array of graphene-corrugated carton. Figure 5 a showed the voltampere characteristics of four sensors on the array. The initial resistance was investgated. As shown in figure $5 \mathrm{~b}$, the resistance of four sensors were similar. Therefore, the manufactured sensor array had better consistency. A $2 \times 2$ sensor array was used to investgate weights of different masses. Figure $5 \mathrm{c}$ showed the weights with test masses of $5 \mathrm{~g}, 10 \mathrm{~g}$, and $20 \mathrm{~g}$. Figure $5 \mathrm{~d}$ showed the test results (figure $5 \mathrm{~d}$ ). Sensors in the array had corresponding resistance responses to weights of different masses placed, and can identify the distribution and mass of objects. Put a school badge and a U disk on the array for identification. Figure 5e and g were test picture. The test results were shown in Figure $5 \mathrm{f}$ and $\mathrm{h}$. The school badge was placed on the upper two units of the sensor array. In the result, the upper two units had resistance responses and were consistent, while the lower two units have no object placed, and the resistance response were zero. The location of the $U$ disk can also be obtained through the results.

Corrugated boxes can be used for safety warnings in the laboratory for its easy to obtain and the preparation process was simple. As shown in Figure 5 i-l, different molds were designed to put the corrugated box in it, and print electrodes on the bottom to contact the bulb. When the edge of the switch was accidentally touched, the resistance of the corrugated box became smaller, and the circuit resistance became smaller, and the brightness of the bulb changed, which reminded people to pay attention to the switch. When the touched carton cannot be used due to stress and deformation, the module can be directly taken out and replaced with a new module to realize a detachable and sustainable switch alarm. 


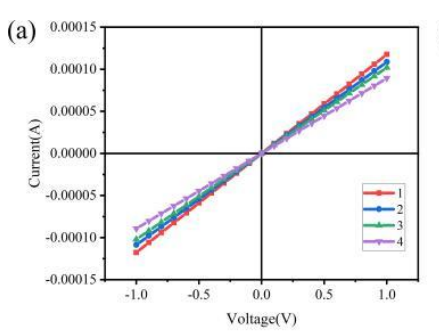

(e)

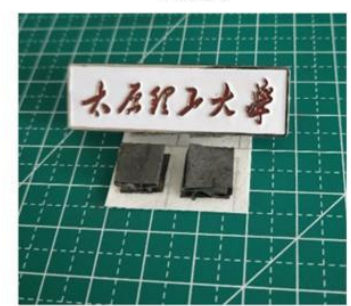

(g)

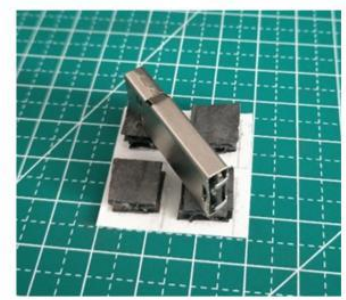

(b)

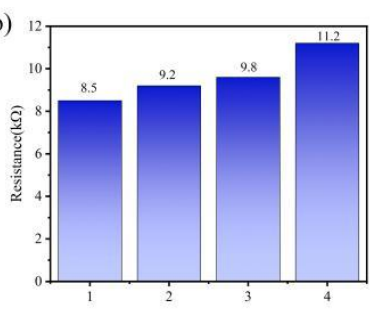

(f)

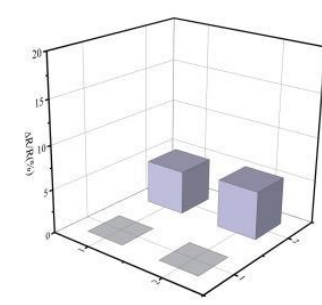

(h)

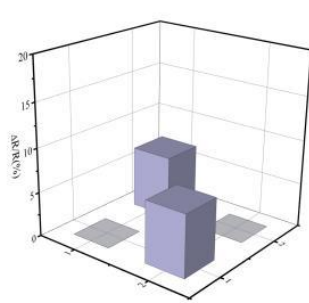

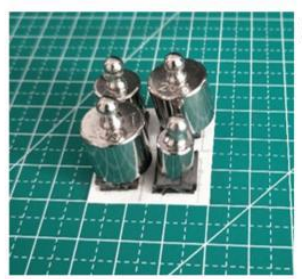

(i)

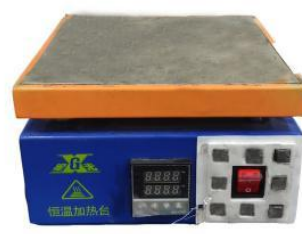

(k)

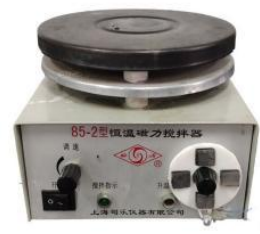

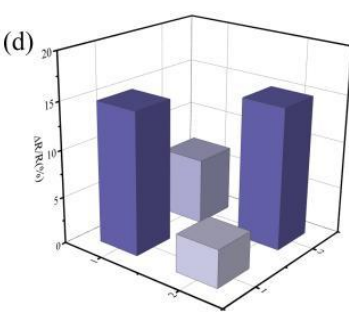

(j)

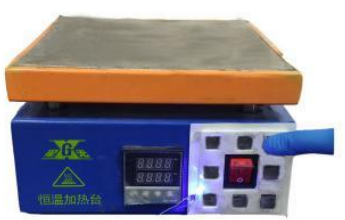

(1)

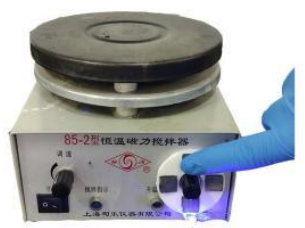

Figure 5. Graphene-corrugated carton array test. Result diagram of (a) I-V characteristic test and (b) resistance in four modules of the $2 * 2$ array. Physical test drawings of (c)different mass weights, (e)school emblem and (g)U disk. Results diagram of (d)different mass weights, (f)school emblem and (h) USB flash disk. Physical picture of placing removable corrugated box array on (i) heating table and (k) magnetic stirrer for safety identification. Physical view of the changing brightness of the bulb (j)on the heating table and (I) magnetic stirrer when pressed with a finger. Owing to its higher sensitivity, a $2 \times 2$ array was prepared with 7 layers paper-based sensors, and the results were shown in Figure 6. The pressure response characteristics on the four modules were shown in Figure 6a, which showed that the sensitivities of the 4 modules were similar and the array test can be performed. Using $5 \mathrm{~g}, 10 \mathrm{~g}, 20 \mathrm{~g}$ weights for tested, the result was shown in Figure 6c, which showed that the array can achieve resistance response to different mass weights, and compared with the corrugated box array results. The array had a more obvious response to the weight test. Placing the same school badge and U disk on the multi-layer paper-based sensor array, as shown in Figure $6 \mathrm{~d}$ and $\mathrm{f}$, and the result were shown in Figure 6e and g. 

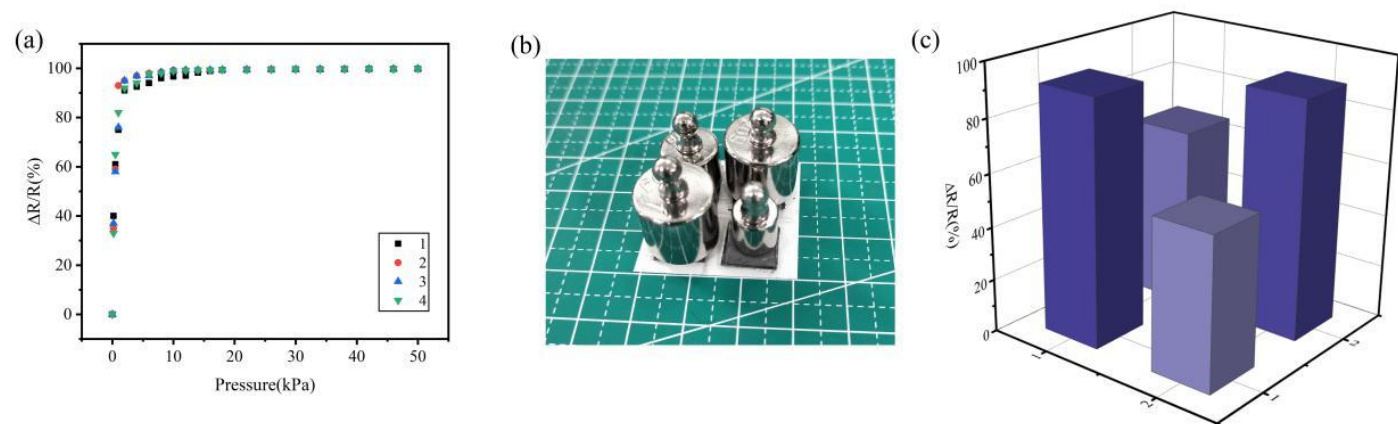

(d)

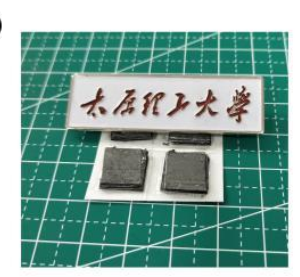

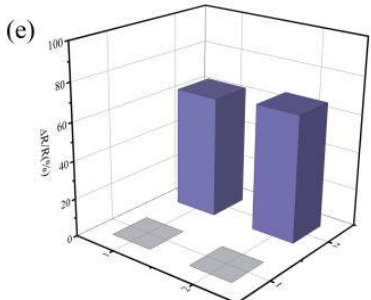

(f)

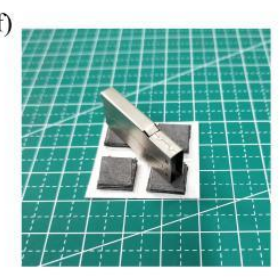

Figure 6. Graphene-7 layers cellulose paper sensor array. (a) Sensitivity test results of four modules on the array. Physical test drawings of (b)different mass weights, (d)school emblem and (f)U disk. Results diagram of (c)different mass weights, (e)school emblem and (g) USB flash disk.

Due to the writing brush can be used to write different patterns on the cellulose paper, the graphene-cellulose paper can be made with different patterns, and the design was more life-like. A small house shape that can detect objects was designed (Figure 7a-d). First, two single-layer graphene-cellulose papers were used to design the pillars and connect two led lights. Lead out the electrode, and glue the electrodes with silver underneath. Then the three layers graphene-cellulose paper was used to design the bottom of the house, the complete path of the stroke circuit, provide voltage. When the beaker was placed on the bottom of the house, the resistance becomes smaller and caused the circuit current to increase and the bulb brighter. A small patch was designed that can be placed on the bottle (Figure 7e-h). When the hand touched the surface of the patch, the contact of the inner three layers of graphene-cellulose paper was increased, and the bulb turned from dark to bright, realized the recognition of cup grabbing. 


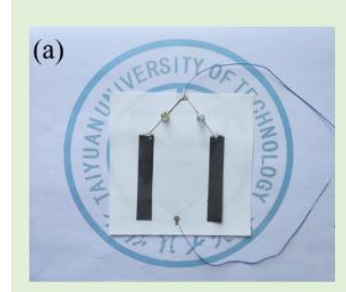

(b)

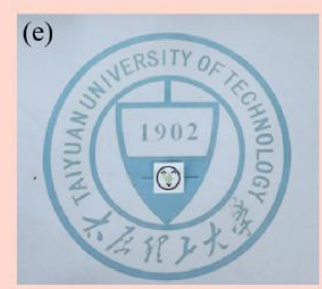

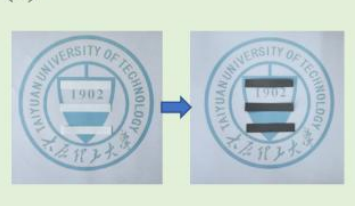

(f)

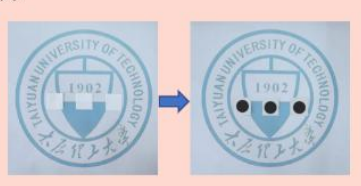

(c)
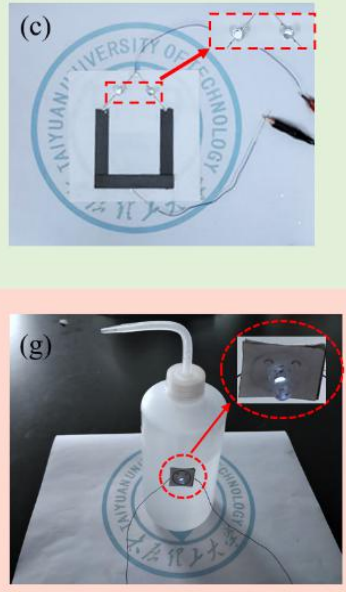

(d)
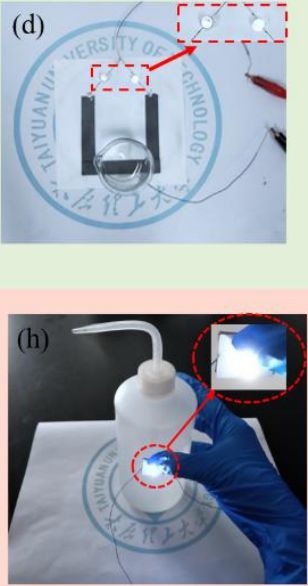

Figure 7 Writable graphene-cellulose paper-based sensor life application scenarios. (a) Two LED lights connected with two pieces of cellulosic paper which written graphene, (b) Use graphene ink to write three blank cellulose paper, (c) Three sheets of graphene are placed on the electrode paper at the bottom using double-sided adhesive tape. The power is connected and the bulb is slightly bright, (d) Using a beaker to press the stacked graphene paper, the light bulb become brighter. (e) A smiley face labeled LED bulb, (f) Use graphene ink to write three blank sheets of cellulose paper with a circular center, (g) Attach the sensor patch to the bottle, connect the power supply, and the bulb will be slightly bright, (h) When the hand grips the bottle, the light bulb gets brighter.

There are many warning lines in life that need people to pay attention to and cannot be crossed, especially under the COVID-19. For that reason, a safety warning line reminder was designed. Figure 8a showed "T" written on cellulose paper, which would be stacked together as shown in Figure 8b. The graphene written on the paper forms a conductive layer between different layers. Then, as shown in Figure 8c, a led bulb was placed on the stacked paper, and the remaining "Y", "U", and "T" were prepared in the same way. With the distance gradually decreases, the warning level gradually rises, and the lights were blue, green, yellow and red in turn. Then four sensors were placed around the cordon, as shown in Figure 8e. When a people steps on sensors, the corresponding light bulb color would light up, and becomes more dazzling as people steps foreward. This system can serve as a reminder to people who have crossed the cordon. 


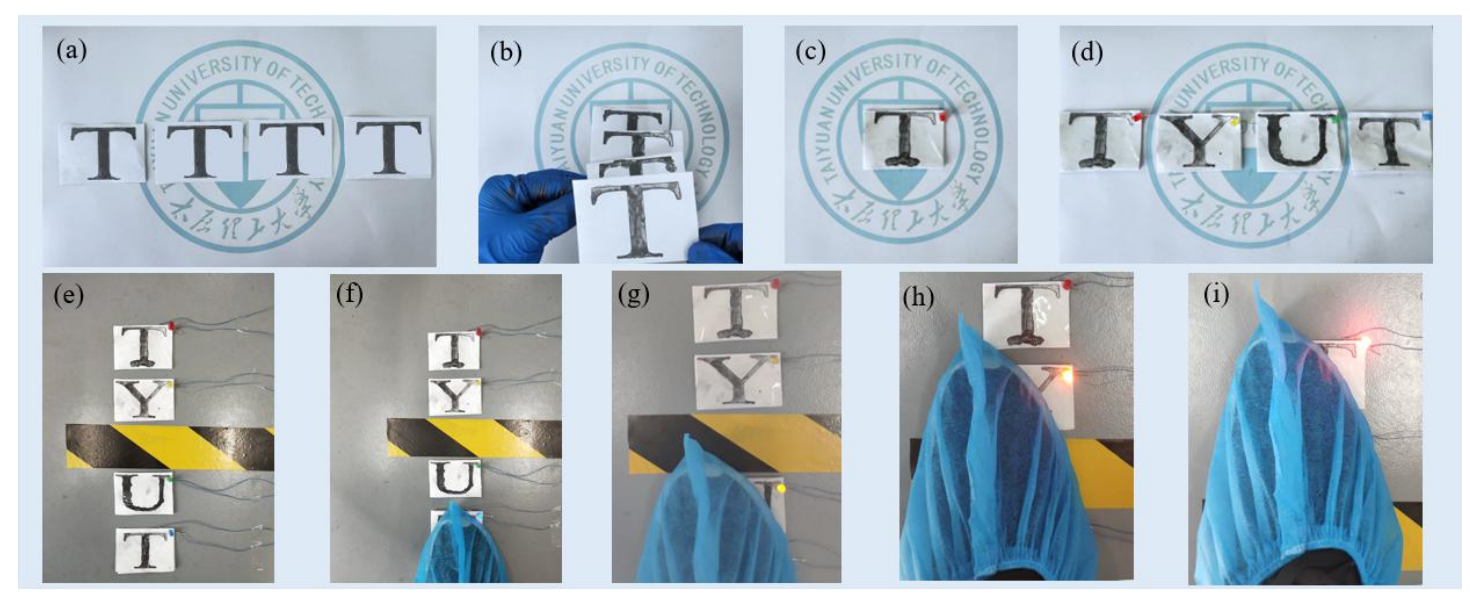

Figure 8 Writable graphene-cellulose paper-based sensor for safety warning. (a) Write "T" on cellulose paper with a brush, (b) Stack the four sheets of paper with the "T" written on them, (c) Connect the LED bulb to the paper with the " $T$ " written on it and wrap it with tape, (d) "T", "Y", "U" and "T" prepared in order according to the above method. (e-i) warning for different step ranges

\section{Conclusion}

In this study, we prepared graphene-based pressure sensors for different layers of cellulose paper and corrugated boxes based on the good permeability of the paper substrate and the good conductivity of graphene. Highly sensitive and repeatable of pressure sensor was realized. The results showed that as the number of layers increases, the sensitivity increase and the response range becomes smaller. The sensitivity of the seven-layer cellulose paper was $44 \mathrm{kPa}^{-1}$, the range was $0 \sim 4 \mathrm{kPa}$ and the response time was $150 \mathrm{~ms}$. In order to compare this layer structure with easy available corrugated carton, a corrugated carton sensor was prepared by soaking method, which has a sensitivity of $8 \mathrm{kpa}^{-1}$, the range was $0 \sim 10 \mathrm{kPa}$, and the response time was $270 \mathrm{~ms}$. The paper-based sensing array with layered structure and high sensitivity was prepared by using writing method and applied to graphic pressure sensing and distance position detection. The corrugated carton was made into a safety reminder device for experimental equipment, and graphene was written on cellulose paper to make a device that can be used in life, and a reminder device for safety cordon. The sensor will play a good safety warning role in daily applications. 
This study was financially supported by National Natural Science Foundation of China (NO.

51975400), the Shanxi University Innovation Project of China (RD1900000640), Excellent

Talents Technology Innovation Program of Shanxi Province of China(201805D211026),

Natural Science Foundation of Shanxi Province (201901D111097), and Beijing Natural

Science Foundation (7202190).

\section{References}

[1] Fu M, Zhang J, Jin Y, Zhao Y, Huang S, Guo C. A Highly Sensitive, Reliable, and High-

Temperature-Resistant Flexible Pressure Sensor Based on Ceramic Nanofibers. Advanced Science. 2020; 7(17): 2000258.

[2] Shu Y, Li C, Wang Z, Mi W, Li Y, Ren. T. A Pressure Sensing System for Heart Rate

Monitoring with Polymer-Based Pressure Sensors and an Anti-Interference Post Processing Circuit. Sensors. 2015; 15(2): 3224-3235.

[3] Tang X, Wu C, Gan L, ZhangT, Zhou T, Huang J, Wang H, Xie C, Zeng D. Multilevel

Microstructured Flexible Pressure Sensors with Ultrahigh Sensitivity and Ultrawide Pressure Range for Versatile Electronic Skins. Small. 2019; 15(10): 804559.

[4] Yang J, Liu Q, Deng Z, Gong M, Lei F, Zhang J, Zhang X, Wang Q, Liu Y, Wu Z, Guo C. Ionic liquid-activated wearable electronics. Materials Today Physics. 2019; 8: 78-85.

[5] Cai F, YiC, Liu S, Wang Y, Liu L, Liu X, Xu X, Wang L. Ultrasensitive, passive and wearable sensors for monitoring human muscle motion and physiological signals. Biosensors \& Bioelectronics. 2016; 77: 907-913.

[6] Ding Y, Xu T, Onyilagha O, Fong H, Zhu Z. Recent Advances in Flexible and Wearable Pressure Sensors Based on Piezoresistive 3D Monolithic Conductive Sponges. ACS Applied Materials \& Interfaces. 2019; 11(7): 6685-6704.

[7] Yang Y, Tao L, Pang Y, Tian H, Ju Z, Wu X, Yang Y, Ren T. An ultrasensitive strain sensor with a wide strain range based on graphene armour scales. Nanoscale. 2018; 10(24): 11524-11530.

[8] Yang H, Xue T, Li F, Liu W, Song Y. Graphene: Diversified Flexible 2D Material for Wearable Vital Signs Monitoring Advanced Materials Technologies. 2019; 4(2): 1800574.

Wearable Pressure Sensor for Physiological Signal Acquisition and Soft Robotic Skin. ACS Applied Materials \& Interfaces. 2017; 9(43): 37921-37928. 
[10] Bauer, S. Flexible Electronics Sophisticated skin. Nature Materials. 2013;12(10): 871872 .

[11] Liang J, Wu J, Huang H, Xu W, Li B, Xi F. Soft Sensitive Skin for Safety Control of a Nursing Robot Using Proximity and Tactile Sensors. IEEE Sensors Journal. 2020; 20(7): 3822-3830.

[12] Tang X, Wu C, Gan L, Zhang T, Zhou T, Huang J, Wang H, Xie C, Zeng D. Multilevel Microstructured Flexible Pressure Sensors with Ultrahigh Sensitivity and Ultrawide Pressure Range for Versatile Electronic Skins. Small. 2019; 15(10):1804559.

[13] Zhang R, Palumbo A, Hader G, Yan K, Chang J, Wang H, Yang E. A Flexible Pressure Sensor With Sandwiched Carpets of Vertically Aligned Carbon Nanotubes Partially Embedded in Polydimethylsiloxane Substrates. IEEE Sensors Journal. 2020; 20(20): 1214612153.

[14] Bilent S, Dinh THN, Martincic E, Joubert PY. Influence of the Porosity of Polymer Foams on the Performances of Capacitive Flexible Pressure Sensors. Sensors. 2019; 19(9): 1968.

[15] Shuai X, Zhu P, Zeng W, Hu Y, Liang X, Zhang Y, Sun R, Wong C. Highly Sensitive Flexible Pressure Sensor Based on Silver Nanowires-Embedded Polydimethylsiloxane Electrode with Microarray Structure. ACS Applied Materials \& Interfaces. 2017; 9(31): 26314-26324.

[16] Han CJ, Park BG, Oh MS, Jung SB, Kim JW. Photo-induced fabrication of Ag nanowire circuitry for invisible, ultrathin, conformable pressure sensors. Journal of Materials Chemistry C. 2017; 5(38): 9986-9994.

[17] Rana V, Gangwar P, Meena JS, Ramesh AK, Bhat KN, Das S, Singh P. A highly sensitive wearable flexible strain sensor based on polycrystalline MoS2 thin film. Nanotechnology. 2020; 31(38): 385501.

[18] Khurana V, Kaur M, Kumar S, Gupta D, Goswamy JK. Multifunctional graphitic tracks on flexible polymer sheet as strain, acoustic vibration and human motion sensor. Measurement. 2019; 146: 9-14.

[19] Yin Z, Yin M, Liu Z, Zhang Y, Zhang A, Zheng Q. Solution-Processed Bilayer Dielectrics for Flexible Low-Voltage Organic Field-Effect Transistors in Pressure-Sensing Applications. Advanced Science. 2018; 5(9): 1701041.

[20] Lin X, Gao S, Fei T, Liu S, Zhao H, Zhang T. Study on a paper-based piezoresistive sensor applied to monitoring human physiological signals. Sensors and Actuators A-Physical. 2019; 292: 66-70. 
[21] Pataniya PM, Sumesh CK, Tannarana M, Zankat CK, Solanki GK, Patel KD, Pathak VM. Flexible paper based piezo-resistive sensor functionalised by 2D-WSe2 nanosheets. Nanotechnology. 2020; 31(43): 435503.

[22] Yang W, Cao R, Zhang X, Li H, Li C. Air-Permeable and Washable Paper-Based Triboelectric Nanogenerator Based on Highly Flexible and Robust Paper Electrodes. Advanced Materials Technologies. 2018; 3(11): 1800178.

[23] Liao X, Zhang Z, Liao Q, Liang Q, Ou Y, Xu M, Li M, Zhang G, Zhang Y. Flexible and printable paper-based strain sensors for wearable and large-area green electronics. Nanoscale. 2016; 8(26): 13025-13032.

[24] Liana DD, Raguse B, Gooding JJ, Chow E. An Integrated Paper-Based Readout System and Piezoresistive Pressure Sensor for Measuring Bandage Compression. Advanced Materials Technologies. 2016; 1(9): 1600143.

[25] Yang T, Mativetsky JM. Paper-Based Mechanical Sensors Enabled by Folding and Stacking. ACS Applied Materials \& Interfaces. 2019; 11(29): 26339-26345.

[26] Guo Y, Zhong M, Fang Z, Wan P, Yu G. A Wearable Transient Pressure Sensor Made with MXene Nanosheets for Sensitive Broad-Range Human-Machine Interfacing. Nano Letters. 2019; 19(2): 1143-1150.

[27] Tao Q, Zhang K, Tian H, Liu Y, Wang D, Chen Y, Yang Y, Ren T. Graphene-Paper Pressure Sensor for Detecting Human Motions. ACS Nano. 2017; 11(9): 8790-8795.

[28] Liu H, Xiang H, Ma Y, Li Z, Meng Q, Jiang H, Wu H, Li P, Zhou H, Huang W. Flexible, Degradable, and Cost-Effective Strain Sensor Fabricated by a Scalable Papermaking Procedure. ACS Sustainable Chemistry \& Engineering. 2018; 6(11): 15749-15755.

[29] Zhang X, Li K, Li H, Fu Y, Fei J. Influence of compound mineral fiber on the properties of paper-based composite friction material. Proceedings of The Institution of Mechanical Engineers Part J-Journal of Engineering Tribology. 2013; 227(11): 1241-1252.

[30] Zhang H, Sun X, Hubbe M, Pal L. Highly conductive carbon nanotubes and flexible cellulose nanofibers composite membranes with semi-interpenetrating networks structure. Carbohydrate Polymers. 2019;22: 115013.

[31] Li Y, Samad YA, Taha T, Cai G, Fu S, Liao K. Highly Flexible Strain Sensor from Tissue Paper for Wearable Electronics. ACS Sustainable Chemistry \& Engineering. 2016;4(8): 4288-4295.

[32] Zhu Z, Zhang H, Xia K, Xu Z. Pencil-on-paper strain sensor for flexible vertical interconnection. Microsystem Technologies-Micro-and Nanosystems-Information Storage and Processing Systems. 2018; 24(8): 3499-3502. 
[33] Liao X, Liao Q, Yan X, Liang, Q, Si H, Li M, Wu H, Cao S, Zhang Y. Flexible and

340 Highly Sensitive Strain Sensors Fabricated by Pencil Drawn for Wearable Monitor. Advanced 341 Functional Materials. 2015; 25(16): 2395-2401.

[34] Zhu J, Song W, Huang R. Modulated Pencil-Drawn U-Shaped Piezoresistive Graphite on

Compound Fibers for Wind Sensing. Journal of Electronic Materials. 2018; 47(11): 65186524.

[35] Jung M, Kim K, Kim B, Cheong H, Shin K, Kwon OS, Park JJ, Jeon S. Paper-Based Bimodal Sensor for Electronic Skin Applications. ACS Applied Materials \& Interfaces. 2017; 9 (32): 26974-26982.

[36] Sekertekin Y, Bozyel I, Gokcen D. A Flexible and Low-Cost Tactile Sensor Produced by Screen Printing of Carbon Black/PVA Composite on Cellulose Paper. Sensors. 2020; 20(10): 2908.

351 [37] Gao L, Zhu C, Li L, Zhang C, Liu J, Yu H, Huang W. All Paper-Based Flexible and 352 Wearable Piezoresistive Pressure Sensor. ACS Applied Materials \& Interfaces. 2019; 11(28): $353 \quad 25034-25042$.

354 [38] Hu M, Cai X, Guo Q, Bian B, Zhang T, Yang J. Direct Pen Writing of Adhesive Particle355 Free Ultrahigh Silver Salt-Loaded Composite Ink for Stretchable Circuits. ACS Nano. 2016; 356 10(1): 396-404.

[39] Lee K, Lee J, Kim G, Kim Y, Kang S, Cho S, Kim S, Kim JK, Lee W, Kim DE, Kang S, Kim D, Lee T, Shim W. Rough-Surface-Enabled Capacitive Pressure Sensors with 3D Touch Capability. Small. 2017; 13 (43): 1700368. [40] Wang F, Mao P, He H. Dispensing of high concentration Ag nano-particles ink for ultralow resistivity paper-based writing electronics. Scientific Reports. 2016; 6: 21398.

[41] Costa JC, Wishahi A, Pouryazdan A, Nock M, Munzenrieder N. Hand-Drawn Resistors, Materials 2018; 4(5): 1700600. 